\title{
Comment l'action publique peut faire toute la différence pour les petites et moyennes entreprises
}

Écrit par : OCDE

Dernière mise à jour : 1 juin 2019

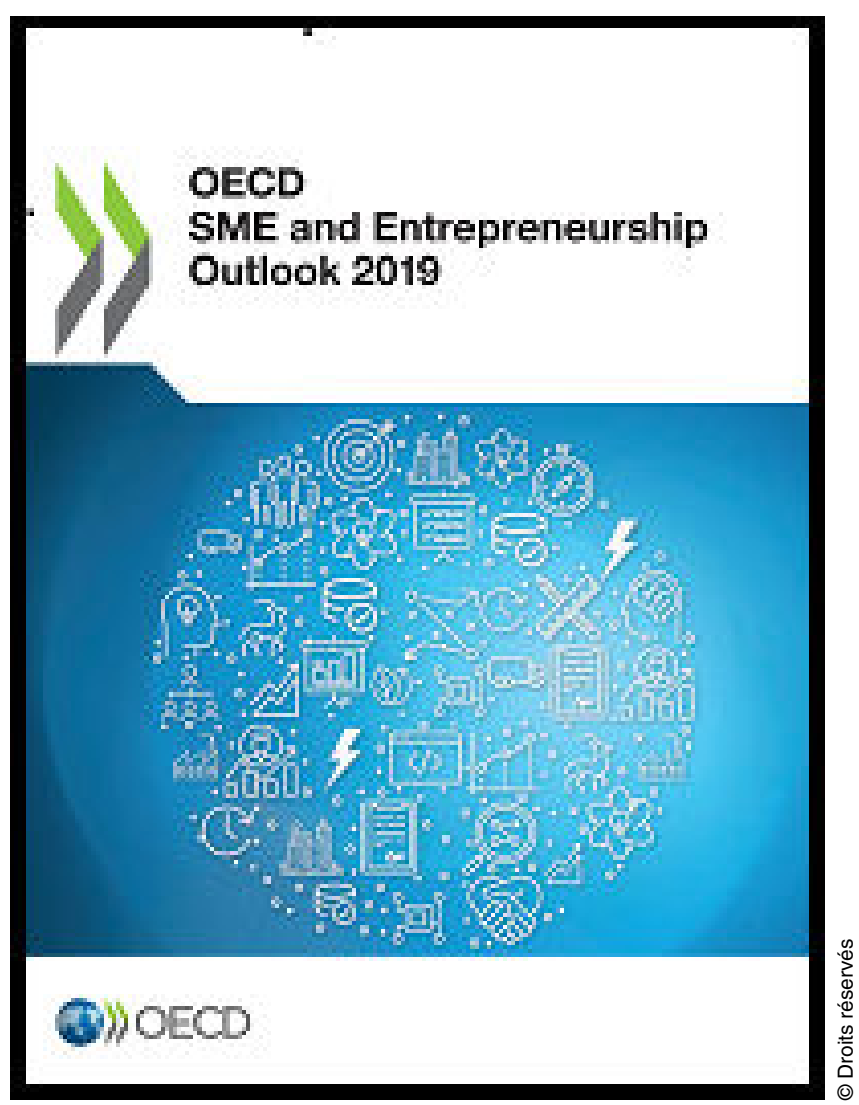

Saviez-vous que presque toutes les entreprises des pays de l'OCDE sont en fait des petites et moyennes entreprises ? Non seulement ces PME représentent $99 \%$ du nombre total d'entreprises, mais elles génèrent également $60 \%$ environ de l'emploi et entre $50 \%$ et $60 \%$ de la valeur ajoutée. Il n'est donc pas étonnant que les responsables de l'action publique les considèrent comme la clé d'une croissance économique durable et inclusive. 
Les PME jouent un rôle déterminant pour que nos économies et nos sociétés puissent s'adapter aux transformations majeures, telles que la mondialisation, le numérique, les pressions environnementales et les changements démographiques.

Les pouvoirs publics partagent souvent des orientations générales communes concernant les PME et l'entrepreneuriat. En revanche, les visions et les approches nationales de la conception et de la mise en œuvre des politiques publiques en faveur des PME diffèrent, certains points de vue divergeant quant aux moyens de libérer le potentiel des PME. Si certains pays ont cherché à intégrer la question des PME dans d'autres programmes d'action, d'autres ciblent spécifiquement cette catégorie d'entreprises au moyen d'instruments taillés sur mesure, allant souvent de pair avec tout un éventail d'actions déployées au niveau local ou sectoriel.

Quelle que soit l'approche stratégique retenue, l'accent est largement mis sur les objectifs suivants : accélérer la diffusion de l'innovation auprès des PME et veiller à ce qu'elles suivent le rythme de la transformation numérique ; associer les PME à l'amélioration des compétences ; développer les réseaux d'innovation et les liens avec les multinationales.

De même, les initiatives des pouvoirs publics visent généralement à instaurer des règles de jeu équitables en encourageant une plus forte concurrence, par exemple, ou en subdivisant les marchés publics en lots de taille plus modeste (allotissement), en simplifiant les procédures et l'accès à la formation, et en favorisant l'adoption de pratiques innovantes dans la passation des marchés publics ou un soutien ciblé à l'adoption de l'innovation dans les PME.

Les gouvernements des pays de l'OCDE ont également entrepris des réformes visant à alléger la charge administrative et fiscale pesant sur les entreprises, mais le rythme des réformes structurelles a marqué le pas ces dernières années, notamment s'agissant des régimes d'insolvabilité et de la possibilité d'offrir d'une seconde chance aux entrepreneurs.

Des défis se profilent à l'horizon. Le moteur des PME a redémarré et dynamisé la création d'emplois depuis 2010, mais les nouveaux emplois ont souvent été créés dans des activités peu productives et peu rémunératrices. Entre 2010 et 2016, près de $90 \%$ des créations nettes d'emplois en France, 75 \% aux États-Unis, et 66 \% en Allemagne et au Royaume-Uni, ont été concentrées dans des secteurs à bas salaires, ce qui a contribué à la stagnation des salaires moyens et au creusement des inégalités.

Certaines PME font course en tête à la frontière de la connaissance : parmi elles, on trouve de grandes championnes de l'innovation et de la productivité, en particulier dans les services spécialisés tels que les activités professionnelles, scientifiques et techniques, où la taille ne joue pas un rôle aussi décisif. Pourtant, de nombreuses PME calent lorsqu'il s'agit d'investir dans les compétences, les

2 www.oecdobserver.org I Comment l'action publique peut faire toute la différence pour les petites et moyennes entreprises 
technologies et l'innovation qui seraient nécessaires pour doper les salaires et la productivité.

En particulier, les PME sont à la traîne dans le domaine du numérique. Des mesures des pouvoirs publics sont nécessaires pour qu'elles puissent aller plus de l'avant dans l'adoption des technologies numériques et dans la protection de leurs données, et être mieux préparées à faire face aux menaces qui planent sur leur cybersécurité. Les PME doivent redoubler d'efforts pour améliorer les compétences nécessaires à la gestion de leur transformation numérique et mobiliser davantage leurs salariés dans des formations au monde du travail numérique. Les progrès accomplis récemment doivent être consolidés, qu'il s'agisse de la participation à des programmes d'enseignement professionnel ou de la réduction du déficit de formation par rapport aux grandes entreprises, afin de combler le fossé existant en matière de compétences.

Les politiques publiques devraient aider les PME à tirer parti des nouvelles possibilités offertes par la révolution de la production en s'emparant de l'analytique des données massives et de l'intelligence artificielle pour améliorer la différenciation et la personnalisation de leurs produits et services. La montée en puissance du numérique peut aider les PME à s'intégrer sur les marchés mondiaux et dans les chaînes de valeur mondiales. L'internationalisation est un autre moyen important d'accroître la productivité et les salaires des PME. En fait, les chiffres montrent que les écarts de salaires des PME par rapport aux grandes entreprises, qui rémunèrent généralement leurs salariés $20 \%$ de plus que les petites entreprises, sont plus faibles pour les PME qui exportent. 


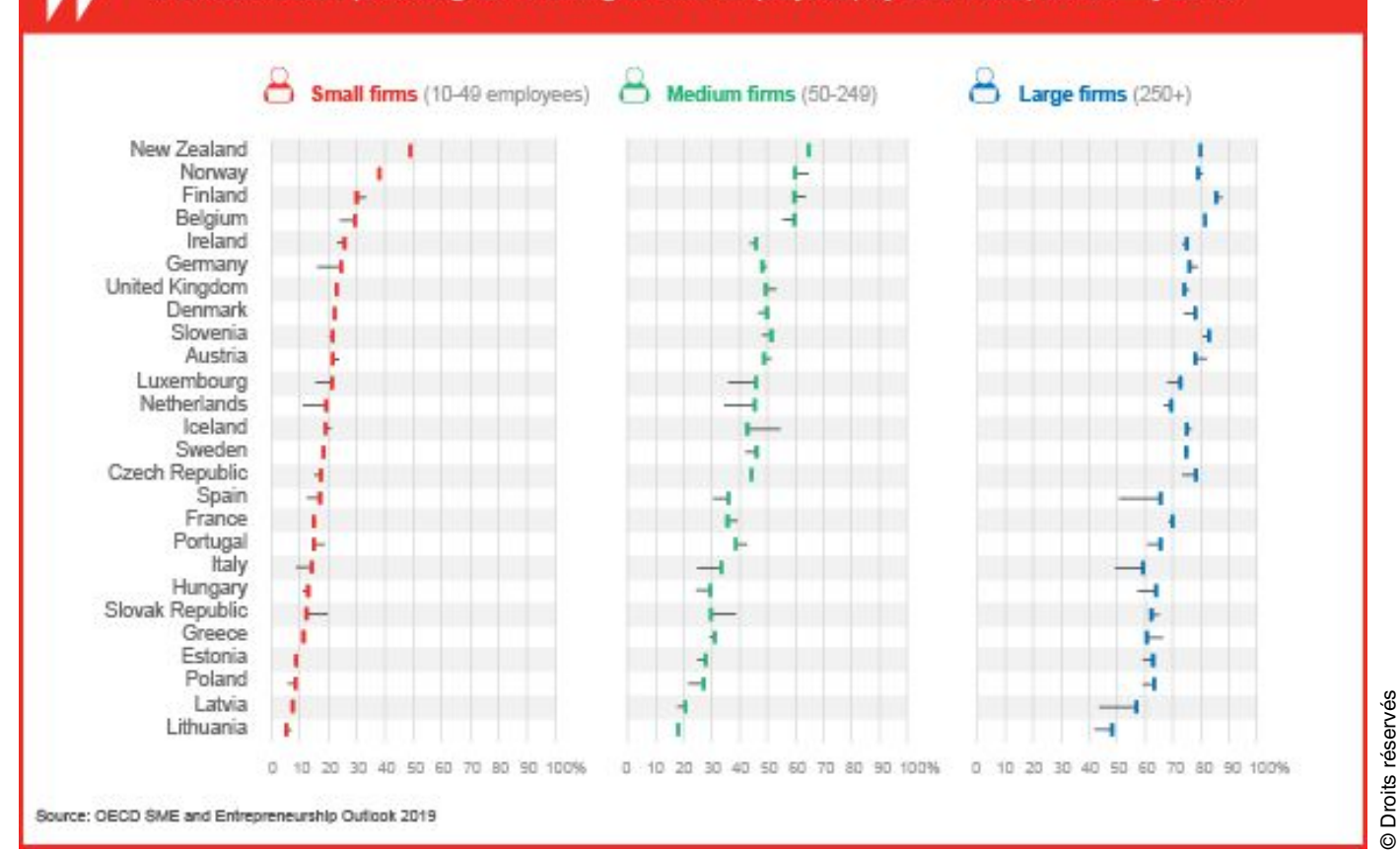

OCDE

Des mesures s'imposent dès maintenant pour permettre aux PME de tirer le meilleur parti des nouvelles opportunités qui se dessinent, mais également pour qu'elles soient mieux préparées en cas de nouveau ralentissement économique. En fait, à mesure que les tensions commerciales s'accentuent et que les conditions sur les marchés se durcissent pour les PME, la marge de manoeuvre dont elles disposent pour profiter de la transformation numérique et de la mondialisation risque de se réduire..

Pour une action efficace, les pouvoirs publics doivent prendre en compte la grande diversité des PME. Il conviendrait également de s'attaquer à la multitude des facteurs qui peuvent influer sur l'environnement des entreprises et relèvent de multiples domaines d'action, de la réglementation aux infrastructures, en passant par la concurrence et la fiscalité, sans parler des niveaux de gouvernance, national et infranational, des politiques publiques.

Nombre de ces questions sont traitées dans les Perspectives sur les PME et l'entrepreneuriat. Les PME peuvent nous aider à atteindre de nouveaux sommets, et grâce à la coopération internationale et à des données plus nombreuses sur les bonnes pratiques, les responsables de l'élaboration des politiques publiques peuvent aider les PME et les entrepreneurs à jouer plus pleinement leur rôle dans la construction de sociétés plus résilientes, plus durables et plus inclusives.

@L'Observateur de l'OCDE, juin 2019 


\section{Références}

OCDE (2019), OECD SME and Entrepreneurship Outlook 2019 («Perspectives de l'OCDE sur les PME et l'entrepreneuriat $2019 »)$, Éditions OCDE, Paris http:// dx.doi.org/https://doi.org/10.1787/34907e9c-en 